\title{
Evidence for TeV gamma ray emission from Cassiopeia $\mathrm{A}$
}

F. Aharonian ${ }^{1}$, A. Akhperjanian ${ }^{7}$, J. Barrio ${ }^{3}$, K. Bernlöhr ${ }^{1}$, H. Börst ${ }^{5}$, H. Bojahr ${ }^{6}$, O. Bolz ${ }^{1}$, J. Contreras $^{3}$, J. Cortina ${ }^{2}$, S. Denninghoff ${ }^{2}$, V. Fonseca ${ }^{3}$, J. Gonzalez ${ }^{3}$, N. Götting ${ }^{4}$, G. Heinzelmann ${ }^{4}$, G. Hermann ${ }^{1}$, A. Heusler ${ }^{1}$, W. Hofmann ${ }^{1}$, D. Horns ${ }^{4}$, A. Ibarra ${ }^{3}$, C. Iserlohe ${ }^{6}$, I. Jung ${ }^{1}$, R. Kankanyan ${ }^{1}$, M. Kestel ${ }^{2}$, J. Kettler ${ }^{1}$, A. Kohnle ${ }^{1}$, A. Konopelko ${ }^{1}$, H. Kornmeyer ${ }^{2}$, D. Kranich ${ }^{2}$, H. Krawczynski ${ }^{1,9}$, H. Lampeitl ${ }^{1}$,

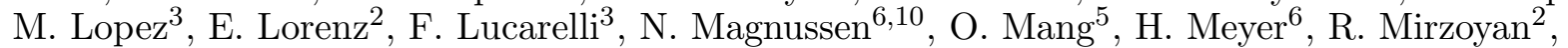

A. Moralejo ${ }^{3}$, E. Ona ${ }^{3}$, L. Padilla ${ }^{3}$, M. Panter ${ }^{1}$, R. Plaga ${ }^{2}$, A. Plyasheshnikov ${ }^{1,8}$, J. Prahl $^{4}$, G. Pühlhofer ${ }^{1}$, G. Rauterberg ${ }^{5}$, A. Röhring ${ }^{4}$, W. Rhode ${ }^{6}$, G. P. Rowell ${ }^{1}$, V. Sahakian ${ }^{7}$, M. Samorski ${ }^{5}$, M. Schilling $^{5}$, F. Schröder ${ }^{6}$, M. Siems ${ }^{5}$, W. Stamm ${ }^{5}$, M. Tluczykont ${ }^{4}$, H. J. Völk ${ }^{1}$, C. A. Wiedner ${ }^{1}$, and W. Wittek ${ }^{2}$

1 Max-Planck-Institut für Kernphysik, Postfach 103980, 69029 Heidelberg, Germany

2 Max-Planck-Institut für Physik, Föhringer Ring 6, 80805 München, Germany

3 Universidad Complutense, Facultad de Ciencias Físicas, Ciudad Universitaria, 28040 Madrid, Spain

4 Universität Hamburg, II. Institut für Experimentalphysik, Luruper Chaussee 149, 22761 Hamburg, Germany

${ }^{5}$ Universität Kiel, Institut für Experimentelle und Angewandte Physik, Leibnizstraße 15-19, 24118 Kiel, Germany

6 Universität Wuppertal, Fachbereich Physik, Gaußstr. 20, 42097 Wuppertal, Germany

7 Yerevan Physics Institute, Alikhanian Br. 2, 375036 Yerevan, Armenia

8 On leave from Altai State University, Dimitrov Street 66, 656099 Barnaul, Russia

9 Now at Yale University, PO Box 208101, New Haven, CT 06520-8101, USA

10 Now at IFAE, Unversitat Autònoma de Barcelona, Spain

Received 23 November 2000 / Accepted 23 January 2001

\begin{abstract}
HEGRA Stereoscopic Cherenkov Telescope System to observe the supernova remnant Cassiopeia A. TeV $\gamma$-ray emission was detected at the $5 \sigma$ level, and a flux of $\left(5.8 \pm 1.2_{\text {stat }} \pm 1.2_{\text {syst }}\right) 10^{-9} \mathrm{ph} \mathrm{m}^{-2} \mathrm{~s}^{-1}$ above $1 \mathrm{TeV}$ was derived. The spectral distribution is consistent with a power law with a differential spectral index of $-2.5 \pm 0.4_{\text {stat }} \pm 0.1_{\text {syst }}$ between 1 and $10 \mathrm{TeV}$. As this is the first report of the detection of a TeV $\gamma$-ray source on the "centi-Crab" scale, we present the analysis in some detail. Implications for the acceleration of cosmic rays depend on the details of the source modeling. We discuss some important aspects in this paper.
\end{abstract}

Key words. ISM: supernova remnants - ISM: individual objects: Cassiopeia A - cosmic rays - gamma rays: observations

\section{Introduction}

Supernova remnants (SNRs) are widely believed to be the acceleration sites for cosmic rays $(\mathrm{CR})$ - ions as well as electrons - up to particle energies of at least $10^{15} \mathrm{eV}$ (see Völk 1997; Baring 2000, for recent reviews). Astronomical evidence for this theory, i.e. via identification of sources, is based on the detection of accompanying $\gamma$-rays which are produced in or nearby the source.

Hadronic CR produce $\gamma$-rays in collisions with gas, mainly via $\pi^{0}$-decay. At $\mathrm{GeV}$ energies, SNR source candidates compete with the diffuse Galactic $\gamma$-ray background (Esposito et al. 1996). Optimum sensitivity is currently

Send offprint requests to: G. Pühlhofer,

e-mail: Gerd.Puehlhofer@mpi-hd.mpg.de expected at TeV energies (Drury et al. 1994); nevertheless, a clean detection is lacking.

Hard synchrotron X-ray spectra of several SNRs, including SN 1006 (Koyama et al. 1995), SNR RX J1713.73946 (Koyama et al. 1997), and Cassiopeia A (Cas A) (Allen et al. 1997; Favata et al. 1997), have been interpreted as evidence for non-thermally accelerated electrons up to $\approx 100 \mathrm{TeV}$. Also, $\mathrm{TeV} \gamma$-rays are generated from electron populations by bremsstrahlung and by inverse Compton (IC) upscattering of ambient soft photons, e.g. from the microwave background. Observations of $\mathrm{TeV}$ photon emission from SN 1006 and SNR RX J1713.7-3946, reported by the CANGAROO experiment (Tanimori et al. 1998; Muraishi et al. 2000), have been interpreted in this 
framework, although a hadronic origin should not be excluded (Aharonian \& Atoyan 1999; Berezhko et al. 1999).

Cas A is the brightest shell-type SNR that is accessible to the HEGRA experiment. "Brightest" applies to the radio band (Atoyan et al. 2000a, and references therein) as well as to non-thermal X-rays (Allen et al. 1999). The remnant results from the youngest known Galactic supernova which dates from around 1680. Its distance is estimated at $3.4 \mathrm{kpc}$ (Reed et al. 1995). The images clearly reveal the shell-type nature of the remnant. Only recent high resolution X-ray maps from the Chandra satellite (Hughes et al. 2000) indicate a central object. The progenitor of Cas A was probably a Wolf-Rayet star, as discussed in Fesen \& Becker (1991) and Iyudin et al. (1997). Its initial mass is estimated to be $\approx 30 M_{\odot}$. The supernova blast wave is expanding into a wind bubble and shell system from the previous wind phases of the progenitor star, which plays an important role in the modeling of the shock acceleration of CR in such remnants (Berezhko \& Völk 2000a). At TeV energies, upper limits have been given by Whipple (Lessard et al. 1999) and CAT (Goret et al. 1999). Nevertheless, detectable flux levels can be expected both from electron emission and the hadronic channel, as we will discuss in Sect. 4.

In this paper, we report the results obtained with the HEGRA Stereoscopic Cherenkov Telescope System. A preliminary analysis, based on parts of the data, was presented in Pühlhofer et al. (1999b) and already revealed evidence for $\mathrm{TeV} \gamma$-ray emission. A summary of the observations and analysis results is given in the next section. Section 3 deals in more detail with the analysis procedure. Finally, in Sect. 4 we interpret our results in the context of model predictions for the $\mathrm{TeV} \gamma$-emission from Cas A.

\section{Observations and summary of the analysis results}

The HEGRA Stereoscopic Cherenkov Telescope System (Konopelko et al. 1999b) is located on the Roque de los Muchachos on the Canary Island La Palma, at $2200 \mathrm{~m}$ above sea level. It consists of 5 identical telescopes (CT 2CT 6), which operate in coincidence for stereoscopic detection of air showers induced by primary $\gamma$-rays in the atmosphere.

Observations were performed in the summer months of 1997, 98 and 99. Cas A can be observed from the HEGRA site at zenith angles of $29^{\circ}$ or larger. The average zenith angle was $32^{\circ}$; observations typically did not go beyond $40^{\circ}$. Hence, the data set provides a $\gamma$-shower peak detection energy of $1 \mathrm{TeV}$ for Crab-like spectra (Konopelko et al. 1999b). Data were taken with 3, 4, and 5 active telescopes. Data cleaning consists of bad weather rejection and exclusion of telescopes with technical problems. Data from the telescope CT 2, which was included last into the system and was still being tuned, were excluded from this analysis. The cleaned data set contains 232 hours of observations.

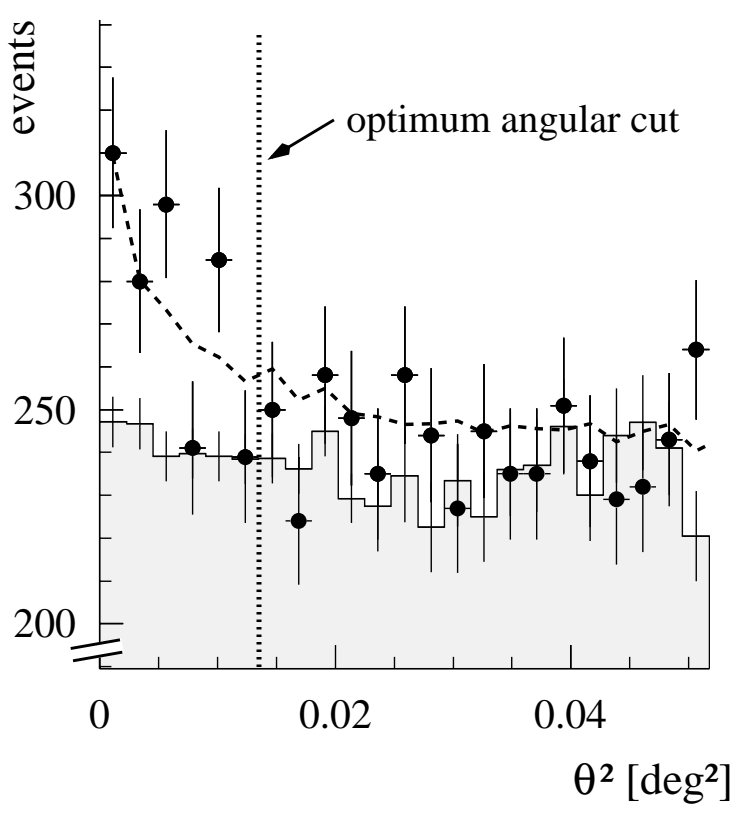

Fig. 1. Dots: number of events vs. the squared angular distance to the position of Cas A. Shaded histogram: background estimate; up to $\theta^{2}=0.0225^{\circ 2}$, data from 7 control regions is used. Therefore, the statistical error of the background estimate is much smaller than the error of the source distribution. The dashed line shows Crab excess events, measured at similar zenith angles, scaled down to $3.3 \%$, and superimposed on a flat background. The vertical dotted line indicates the position of the optimum angular cut

Shower images from a telescope are accepted in the analysis if they contain at least 40 photoelectrons. The particle direction is determined using a stereoscopic reconstruction algorithm described in Hofmann et al. (1999). The data set contains $2.110^{6}, 2.510^{6}$, and $2.910^{6}$ events, for 2,3 , and 4 views available in an event, respectively. Events are accepted within a circle of $1^{\circ}$ radius from the center of the field of view (FOV). Without filtering, virtually all events - coming from the source direction as well as from the surrounding sky region - are induced by hadronic CR's. Candidates for $\gamma$-rays are selected against this background using a cut on the image shape parameter mean scaled width ( $m s w)$ (Aharonian et al. 1999) of $0.5<m s w<1.1$.

In Fig. 1, the number of events is plotted vs. the angular distance to the position of Cas A. The background which remains after the shape cut is estimated using seven control regions in the FOV. The optimum angular cut is derived from Crab and Mrk501 data. The excess significance, calculated after $\mathrm{Li} \& \mathrm{Ma}$ (1983), is $4.9 \sigma$ for this straightforward evaluation.

The photon flux and energy spectrum of Cas A is derived by comparison with a large Crab data sample (Aharonian et al. 2000a), taken between 1997 and 2000. For the spectral analysis, we use the energy reconstruction method described in Aharonian et al. (1999). The spectral distribution is comparable with the distribution measured for the Crab nebula. Under the assumption of a power 
law spectrum $\mathrm{d} F_{\gamma} / \mathrm{d} E \propto E^{\alpha}$ from 1 to $10 \mathrm{TeV}$, we derive a differential spectral index of $\alpha=-2.5 \pm 0.4_{\text {stat }} \pm 0.1_{\text {syst }}$. The flux is $3.3 \%$ of the Crab flux, this corresponds to $F(E>1 \mathrm{TeV})=\left(5.8 \pm 1.2_{\text {stat }} \pm 1.2_{\text {syst }}\right) 10^{-9} \mathrm{ph} \mathrm{m}^{-2} \mathrm{~s}^{-1}$.

\section{Analysis details}

In this paper, we present for the first time evidence for a TeV signal from a source on the "centi-Crab" flux scale, which was obtained after very deep observations made over a three year period. Therefore, some analysis details will be explained here.

The data sample is background-dominated, even after strong background suppression cuts. Control of systematic effects in background determination is therefore far more important than in the case of strong $\mathrm{TeV} \gamma$-ray sources, like the Crab nebula (Aharonian et al. 2000a) or Mrk 501 (Aharonian et al. 1999); Sect. 3.1 deals with the relevant issues. Strong background suppression cuts are needed to obtain a significant signal from Cas A; Sects. 3.2 and 3.3 describe the corresponding procedures. The performance of the HEGRA system changed over the observation time, and in Sect. 3.4 the necessary calibration is discussed. For the determination of the flux and the spectrum of Cas A, we apply the same calibration and cut procedure to a Crab reference data sample, and compare both $\gamma$-ray samples (Sect. 3.5). In this analysis, Cas A is treated as a $\mathrm{TeV} \gamma-$ ray point source; Sect. 3.6 deals with the topic of source localization and extension.

\subsection{Background estimation}

The HEGRA system reveals a nearly flat acceptance for shower directions over the FOV of $2^{\circ}$ diameter, while $95 \%$ of the signal of a point source is contained in a region with a diameter of 0.8 around the source ${ }^{1}$. Therefore we observe point sources in the so-called wobble mode, where the source is displaced \pm 0.5 in declination from the center of the FOV, the sign being reversed every 20 or 30 min. The background can hence be estimated from simultaneously gathered data, and systematic effects in the background estimation due to weather or performance changes essentially cancel out.

Variations of background rates across the FOV are caused by slight acceptance changes, see e.g. Pühlhofer et al. (1999a), and possibly by differences in night sky light noise. For the analysis here, we use seven nonoverlapping control regions centered on a circle of radius 0.5 around the center of the FOV. The setup is sketched in Fig. 2, upper panel. This method has the following characteristics:

(a) It provides better statistics than in the standard case using only one opposite region; the background control area is seven times larger than the signal area;

(b) The regions view different sky areas, closer and

\footnotetext{
1 This is essentially true also in the case of slightly extended sources such as possibly Cas A, see also Sect. 3.6.
}
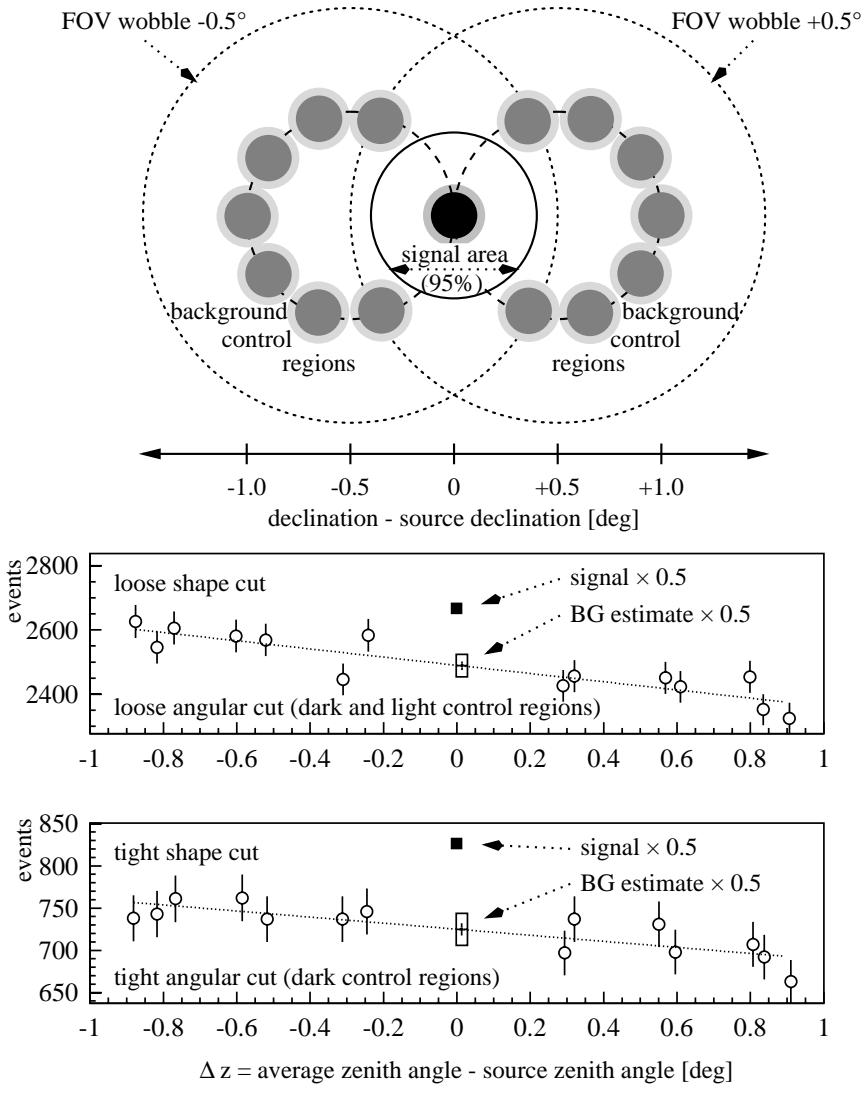

Fig. 2. Upper panel: background control region setup. The dotted circles indicate the FOV, which is alternatingly shifted by plus or minus 0.5 in declination relative to the source position. The solid circle contains $95 \%$ of the signal in case of a point source. Middle and lower panels: Events per control region, as a function of the average zenith angle in the control region with respect to the source zenith angle. The shape cuts are defined in Sect. 3.2. In the middle panel, a loose shape cut and a loose angular cut of $\theta<0.15$ are used. In the lower panel, a tight shape cut and a tight angular cut of $\theta<0.12$ are applied. Each open circle corresponds to one background control region, either in wobble $-0.5\left(\Delta z<0^{\circ}\right)$ or $+0^{\circ} .5$ mode $\left(\Delta z>0^{\circ}\right)$. As the background estimate for the source region, the average over all control regions is used; the value is shown as the small centered cross. The open box indicates the expected $1 \sigma$ background fluctuation range. The filled square shows the number of events in the source region, normalized by a factor 0.5 , since both the wobble +0.5 and -0.5 observing modes contribute to the signal. The statistical significance for the excess in the lower panel is $4.9 \sigma$

further away from the source, and can be cross-checked for sky noise influence (see Fig. 2, middle and lower panels);

(c) The change in radial acceptance is fully taken into account;

(d) Any constant gradient in the background distribution is compensated for; the most significant contribution is due to a zenith angle dependence of the event rate, as can be seen in Fig. 2, middle and lower panels. 
As the background estimate, the average over all control regions is used. A systematic error in the background estimation can possibly be caused by higher-order FOV inhomogeneities. The background levels of the control regions which are closest to the source position deviate by less than $2 \%$ from that estimated for the source location; we consider therefore the systematic error of the background estimation to be below $2 \%$.

In the $\theta^{2}$-distribution (Fig. 1), the background estimation is produced by a properly normalized overlay of all control regions. Note however that the setup described above only works at $0 \leq \theta^{2} \leq 0.0225^{\circ 2}$. Above $0.0225^{\circ 2}$, the background is estimated from adjacent control regions (not shown in Fig. 2), which (I) have only twice the area than the signal region, and (II) cannot fully take the radial acceptance change into account. However, this exclusively affects the right part of Fig. 1, but no other aspect of this analysis.

\subsection{Shape cuts}

A cut on the image parameter mean scaled width ( $m s w$ ) is applied to reduce the background induced by charged CRs (Aharonian et al. 1999). The scaled width is the measured width of an event seen by one telescope, relative to the expected value for a $\gamma$-shower with the given measured distance and image amplitude. By averaging over all telescopes included in an event, one obtains the mean scaled width. The $\gamma$-ray distribution peaks at 1 ; its rms width is approx. 0.1. Charged CR, on the other hand, produce more diffuse images, hence the background distribution peaks (with a much broader distribution) at 1.4-1.55; the exact value changes with zenith angle. The $\gamma$-ray expectation values are usually determined by shower and detector simulations (Konopelko et al. 1999b).

Analyses which focus on the reduction of systematic influences of the background suppression on the $\gamma$ ray efficiency $\kappa_{\gamma}$, use a loose cut of $0.5<m s w<1.2$ $\left(\kappa_{\gamma}=95 \%\right)$, e.g. Aharonian et al. (1999). In the search for faint sources, optimum sensitivity is obtained by a tight cut of $0.5<m s w<1.1$, with an expected $\gamma$-ray efficiency of $\kappa_{\gamma}=80 \%$. Since this cut is more dependant on the exact location of the $\gamma$-ray peak in the mean scaled width distribution, the expectation values were refined to match the quasi background-free $\gamma$-ray distribution of the Mrk 501 data taken in 1997 (Aharonian et al. 1999). The $\gamma$-ray mean scaled width distribution is thereby shifted by $3.5 \%$, to be now exactly centered at 1 . This was verified with our calibration source, the Crab nebula.

In addition, since Crab is not permanently observable, we use the survival probability for background events (typically $\kappa_{\mathrm{BG}}=8 \%$ for the tight, and $16 \%$ for the loose shape cut, at the given zenith angle range) as an indicator for the stability of $\kappa_{\gamma}$. From background control regions in the FOV,$\kappa_{\mathrm{BG}}$ can be continuously derived (see Fig. 3 upper panel).
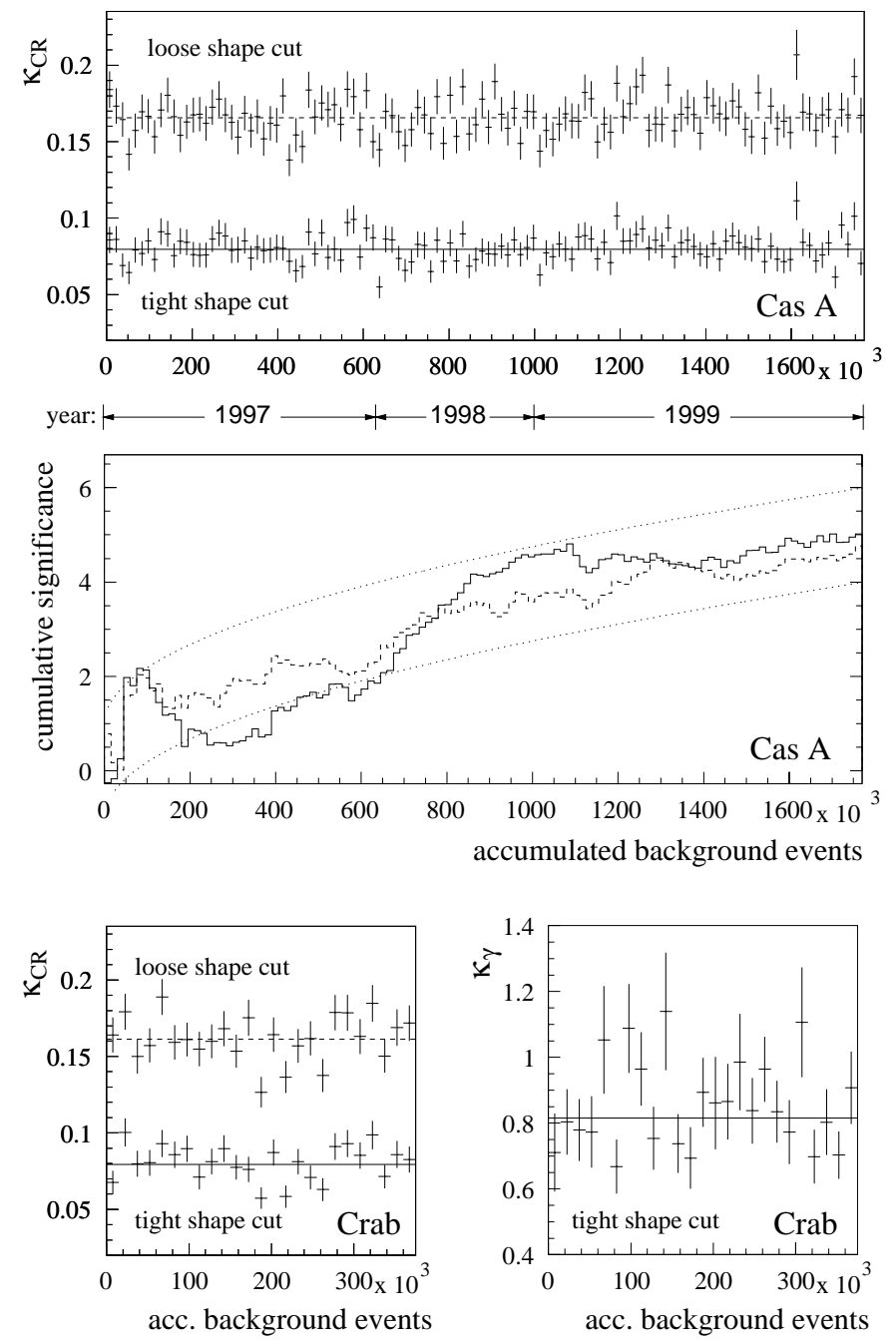

Fig. 3. Upper panel: for the Cas A data set, the survival probability of background events is plotted, broken down into observation time slices; for the "time" axis, the number of accumulated background events in an enlarged FOV of $\varnothing=2.4^{\circ}$ is used. The values are derived from the off source control regions, applying tight and loose shape cuts. Middle panel: the evolution of the Cas A signal with the total number of background events is shown. For a constant signal, the significance should develop as [background events] ${ }^{-1 / 2}$ with a variation of $1 \sigma$; the dotted lines indicate this expected range, assuming a $5 \sigma$ signal at the end of the observation. Lower left panel: for the Crab data set, the same values as in the upper panel are shown; this demonstrates the good agreement of the Cas A and the Crab reference data. Lower right panel: in addition, the $\gamma$ efficiency of the tight shape cut is shown for the Crab data set. The values are derived by comparing the background subtracted sample after the tight shape cut with the background subtracted sample after a cut of $0.5<m s w<1.4$, which rejects virtually no $\gamma$-rays. For all figures, an angular cut of 0.12 was applied

In the context of this analysis, the loose shape cut is only used as an additional consistency check. We note that even if $\kappa_{\gamma}$ changes, this would only induce an error at the flux determination; excess significances are not 
Table 1. Excess significances for different cuts and signal evaluations. All values are derived after tight shape cuts. The number of events is referred to by the symbol \#. Lines A and $\mathrm{B}$ are evaluated with different angular cuts (see text for details); $\alpha$ is the signal to background area normalisation factor ( $\left.\alpha=A_{\text {on }} / A_{\text {off }}\right)$. Line $\mathrm{C}$ corresponds to the full likelihood analysis introduced in the text. $\dagger$ : preliminary value

\begin{tabular}{|c|c|c|c|c|c|}
\hline \multicolumn{6}{|c|}{ "Li \& Ma, Eq. (17) } \\
\hline & \# off & $\alpha$ & \# on & $\# \gamma$ rays & significance \\
\hline $\mathrm{A}$ & 10152 & $1 / 7$ & 1653 & 203 & 4.9 \\
\hline $\mathrm{B}$ & 3165 & $1 / 7$ & 579 & 127 & 5.3 \\
\hline \multicolumn{6}{|c|}{ full likelihood analysis } \\
\hline & \multicolumn{3}{|c|}{ \# complete FOV } & $\# \gamma$ rays & significance \\
\hline $\mathrm{C}$ & \multicolumn{3}{|c|}{103270} & 190 & $5.6^{\dagger}$ \\
\hline
\end{tabular}

affected due to the simultaneous measurement of background events.

\subsection{Angular resolution}

The angular resolution changes with zenith angle and shape cut event selection. The applied stereo reconstruction algorithm (described by Hofmann et al. 1999, algorithm 2) allows the prediction of the resolution on an event basis with an accuracy of $15 \%$.

The standard analysis does not make use of this information; one simply accepts events up to a maximum distance from the source. The given data sample yields a median $\gamma$-ray resolution of 0.09 ; the optimum cut here is 0.12 , which was derived from the point sources Crab and Mrk 501. This cut rejects $40 \%$ of the $\gamma$-rays. For Cas A, the excess significance, following Li \& Ma (1983), amounts to $4.9 \sigma$ (see Table 1 line A).

In a simple approach to make use of the known angular resolution on an event basis, events were divided according to the number of telescopes participating in an event (4, 3, 2 telescopes). The three classes differ significantly both in the angular resolution and the background rejection. Different angular cuts were derived for all classes simultaneously. In order to optimize the sensitivity for the given data sample, the angular cuts were derived such that a data sample which contains $5 \%$ of the Crab $\gamma$-ray flux yields the optimum significance; this training was performed on real data from Crab and Mrk $501^{2}$. The resulting cuts are 0.11 for 4 -telescope events, 0.08 for 3 -telescope events, and the complete rejection of events with only 2 participating telescopes. The excess significance is again derived by a simple counting of signal and background events (Li \& Ma 1983), and amounts to $5.3 \sigma$ (Table 1 line B), in agreement with the expected sensitivity improvement derived from Crab data.

\footnotetext{
2 After tight cuts, signal and background levels start to be at the same order of magnitude (see e.g. Fig. 1). Hence further optimization of the so-called Q-factor $=\kappa_{\gamma} / \sqrt{\kappa_{\mathrm{BG}}}$ does not yield the optimum sensitivity $\left(\kappa_{\gamma}\right.$ and $\kappa_{\mathrm{BG}}$ are the cut survival probabilities for $\gamma$ 's and background events, respectively).
}

In order to fully exploit the stereo information, a maximum likelihood analysis was performed, see e.g. Alexandreas et al. (1993) and references therein. All events after tight shape cuts in the FOV of $\varnothing=2^{\circ}$ are used. The probability density function (PDF) for signal events $P_{\mathrm{s}}$ uses the event-wise predicted 2-dimensional error matrix $C$ for the angular resolution, determined according to the prescription in Hofmann et al. (1999):

$P_{\mathrm{s}}(\mathrm{C} ; \boldsymbol{x})=\frac{1}{2 \pi \sqrt{|\mathrm{C}|}} \exp \left(-\frac{1}{2} \boldsymbol{x}^{T} \mathrm{C}^{-1} \boldsymbol{x}\right)$

where $\boldsymbol{x}$ denotes the FOV vector of the reconstructed event direction. The PDF for background events $P_{\mathrm{b}}$ is calculated with an acceptance function which parametrizes the acceptance of the complete FOV as a function of radius $r$ and zenith angle component $z$ of $\boldsymbol{x}$ :

$P_{\mathrm{b}}(\boldsymbol{x})=P_{\mathrm{b}}(r, z), r=|\boldsymbol{x}|, z=\boldsymbol{x} \cdot \boldsymbol{e}_{\text {zenith }}$.

The likelihood function $\mathcal{L}$ is given by

$\mathcal{L}\left(N_{\mathrm{s}}\right)=\prod\left[r_{i} N_{\mathrm{s}} P_{\mathrm{s}}+\left(N-r_{i} N_{\mathrm{s}}\right) P_{\mathrm{b}}\right]$.

Here, $N$ is the total number of events, and $N_{\mathrm{S}}$ is the number of $\gamma$ candidates coming from the source. We also use an a-priori expected ratio for 4-, 3-, and 2-telescope signal events $r_{i}\left(r_{i=4,3,2} \approx 0.40: 0.35: 0.25\right.$, changing with zenith angle); the values were derived from Crab and Mrk 501 $\gamma$-data. For Cas A, the likelihood ratio test yields a significance of $5.6 \sigma$ (Table 1 line $\mathrm{C}$ ). Our likelihood analysis is still under investigation. The influence of different background parametrizations, obtained from different data sets, was found to be small but not negligible.

\subsection{Performance changes}

The HEGRA system has shown performance changes over the past years which are attributed mainly to the following three effects:

(1) Mirror misalignment, which temporarily lead to changes in the shape cut efficiencies. In this analysis, only Crab data were affected, and data of these periods were rejected. The stability of $\kappa_{\gamma}$ is monitored as discussed in the previous paragraph (see Fig. 3, upper and lower panels).

(2) Aging of mirrors.

(3) Aging of photomultipliers (PMs); the PM gain can be determined independently using laser calibration runs as described in Heß (1998). In spring of 1999, the high voltage of all PMs was increased to compensate for the previous PM gain loss of $15 \%$.

The effects (2) and (3) lead to a change of the light sensitivity of the telescope system. Due to the resulting change in energy threshold, the global sensitivity change can be monitored by the CR trigger rate. The system nominally runs at a trigger rate of $15 \mathrm{~Hz}$ for a 4 -telescope configuration, but the rate temporarily dropped to $70 \%$. Both factors are entered into the calibration procedure to provide an adjusted energy scale. 
For the spectral evaluation of the signal, an extended software threshold is applied, which yields a constant energy acceptance (and also event rate) over time. The extended software threshold emulates the hardware trigger threshold of the telescopes, but on the recalibrated energy scale. It was increased until the resulting $\mathrm{CR}$ event rate was constant over time. Thus, data of Cas A and Crab, which were taken at different observation periods, can be directly compared. Uncertainties in the determination of the energy acceptance function (so called collection area, e.g. Konopelko et al. 1999b), which may arise due to the tight angular and shape cuts, and due to the changing energy threshold, are avoided.

Of course, this method only works at the expense of the number of events. However, the Crab $\gamma$-ray and the background rates show a constant ratio, even without this extended software threshold. For the flux evaluation, it can therefore be omitted in case of Crab-like spectra, in order to have the full statistics available. For compensation of the different detection rates, Cas A and Crab reference data were normalized to each other using total CR event rates.

In Fig. 3 middle panel we show the development of the Cas A signal as a function of the effective exposure time, as determined by the background event rate. Within statistical errors, the excess significance does not deviate from the behaviour which is expected for a steady signal.

\subsection{Flux and energy spectrum}

For flux and spectral evaluation, we compare the data from Cas A with the "standard candle" Crab nebula. The HEGRA Crab data have been evaluated in great detail to derive the Crab flux and spectrum, using data from the full zenith angle range up to $60^{\circ}$ (Aharonian et al. 2000a; Konopelko et al. 1999a). As reference, we use the Crab flux of $\left(\mathrm{d} F_{\gamma} / \mathrm{d} E\right)_{\mathrm{Crab}}=(2.79 \pm 0.02 \pm$ 0.5) $10^{-7}\left(\frac{E}{1 \mathrm{TeV}}\right)^{-2.59 \pm 0.03 \pm 0.05} \mathrm{ph} \mathrm{m}^{-2} \mathrm{~s}^{-1} \mathrm{TeV}^{-1}$ between 1 and $20 \mathrm{TeV}$.

For the determination of the spectral distribution, we apply the energy reconstruction method described in Aharonian et al. (1999), which provides an energy resolution of $20 \%$. The extended software threshold as introduced in Sect. 3.4 is used, in order to obtain adjusted Cas A and Crab reference data samples. The peak detection energy of $\gamma$-rays is derived from Crab data and amounts to $1.4 \mathrm{TeV}$ for this analysis.

Figure 4 upper panel shows the $\gamma$-ray spectra of Cas A and Crab, as well as the background distribution of the Cas A data sample. In Fig. 4, lower panel, the $\gamma$-ray spectrum of Cas A is divided by the Crab $\gamma$-ray spectrum. In the spectral analysis, data below $1 \mathrm{TeV}$ are rejected, since acceptance errors due to the slight difference in the zenith angle distribution of the Cas A and the Crab reference data samples become important.

The fit to a power law spectrum was a priori restricted to the energy range from 1 to $10 \mathrm{TeV}$ : since the
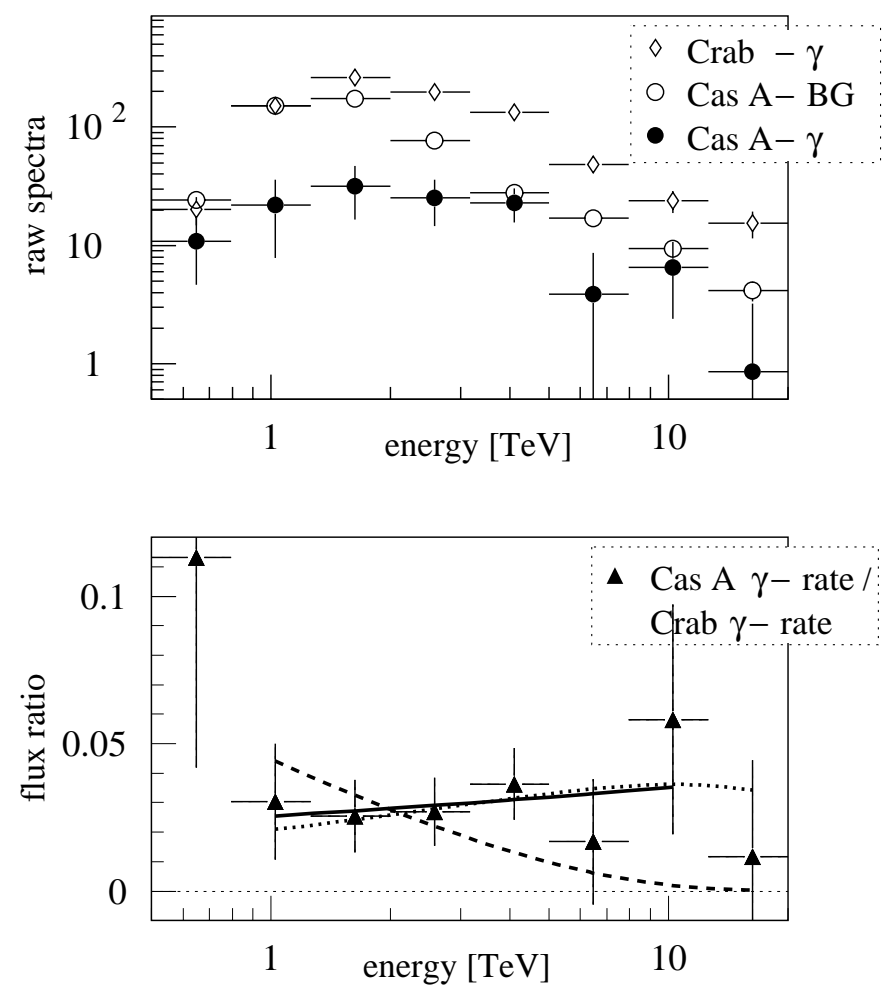

Fig. 4. Differential spectra of the Cas A and Crab data sets. In the upper panel, raw spectra are plotted: $(\diamond)$ Crab on source, background subtracted; $(\bullet)$ Cas A on source, background subtracted; (०) expected background of the Cas A data sample, which is derived from the background control regions and scaled to the source area. The background level of the Crab data set is approx. 5 times lower, due to the shorter exposure time of this reference set. The lower panel shows the ratio of the Cas A and Crab $\gamma$-ray spectra, both normalized by the $\mathrm{CR}$ rate (i.e. essentially by the observation time). The solid line shows the fit of a power law $\mathrm{d} F_{\gamma} / \mathrm{d} E \propto E^{\Delta \alpha}$ with $\Delta \alpha=\alpha_{\text {Cas A }}-\alpha_{\text {Crab }}$. The dotted and dashed curves are fits to the spectral shapes of the hadronic and leptonic emission models which are discussed in Sect. 4

predicted spectra for different model assumptions (see Sect. 4) show cutoffs at higher energies, only a local power law is a reasonable assumption for the comparison between data and predictions; on the other hand, the statistics do not allow much further restriction of the energy range. Assuming for Cas A a power law spectrum $\mathrm{d} F_{\gamma} / \mathrm{d} E \propto E^{\alpha}$, we derive a differential spectral index of $\alpha_{\text {Cas A }}=-2.5 \pm 0.4_{\text {stat }} \pm 0.1_{\text {syst }}$ between 1 and $10 \mathrm{TeV}$ (see solid line in Fig. 4, lower panel).

We also compare the measured spectrum of Cas A to the predicted spectral shapes for hadronic or leptonic emission, as described in Sect. 4. In this test, all data above $1 \mathrm{TeV}$ are included. The fits are shown as the dotted line for the hadronic spectrum, and as the dashed line for the leptonic spectrum. In order to test the compatibility of the data with either model, two statistical tests were applied, a simple $\chi^{2}$-test and a Kolmogorov test (Eadie et al. 1971). The latter is more powerful, since it is also sensitive to a sequence of consecutive deviations of the same sign. 


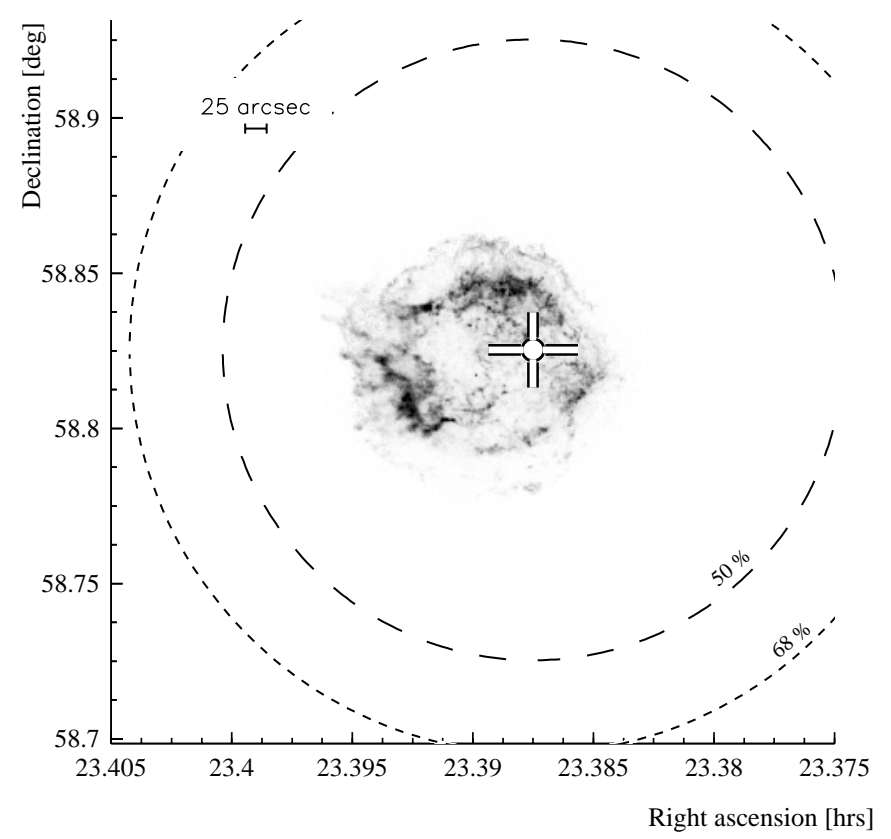

Fig. 5. Cas A (RA 23h 23min 24s, Dec 58 48.'9) in year 2000 celestial coordinates. The cross shows the reconstructed position of Cas A from the $\mathrm{TeV} \gamma$-ray excess, the error bars give the $1 \sigma$ statistical error of the reconstruction. The dashed and dotted circles indicate the $50 \%$ and $68 \%$ single event error, respectively. The systematic pointing uncertainty of the system is 25 arcsec. To indicate the scale, the plot is superimposed on the recent Chandra X-ray image (courtesy of NASA/CXC/SAO)

While the hadronic spectrum has a slightly better chance probability ( $93 \%$ in the $\chi^{2}$-test, $98 \%$ in the Kolmogorov test), the leptonic spectrum has a chance probability of $31 \%$ in the $\chi^{2}$-test and $15 \%$ in the Kolmogorov test and is therefore not ruled out.

We note that a pure background sample would lead to a spectrum which is steeper by $\Delta \alpha \approx-1$. This can be taken as additional evidence that the observed signal from the direction of Cas $\mathrm{A}$ is not due to underestimation of the expected background ${ }^{3}$.

For Cas A's TeV $\gamma$-ray flux, one obtains $3.3 \%$ of the Crab flux; results with and without the extended software threshold are in good agreement. We derive a flux of $F_{\text {Cas A }}(E>1 \mathrm{TeV})=\left(5.8 \pm 1.2_{\text {stat }} \pm\right.$ $\left.1.2_{\text {syst }}\right) 10^{-9} \mathrm{ph} \mathrm{m}^{-2} \mathrm{~s}^{-1}$.

\subsection{Source position}

Cas A's outer shock has a diameter of $5^{\prime}$, which is slightly below the rms angular resolution of the HEGRA telescope system. For the Crab nebula, which has similar extensions, recent studies have shown that resolving this scale

\footnotetext{
${ }^{3}$ Note that the background is a strongly $\gamma$-ray selected sample, and evaluated with a $\gamma$-ray energy reconstruction algorithm. Therefore the background energy spectrum does not represent the charged CR spectrum, but rather the spectrum of $\gamma$-ray-like events (fluctuations of charged CR's, and possibly diffuse $\gamma$-rays and electrons).
}

is marginally out of reach for the system, even with high statistics and a low zenith angle $\gamma$-ray sample (Aharonian et al. 2000b).

For Cas A, the strongest spatial deviation from a point source at the center of the remnant could be caused by a hot spot at the shell, as may be indicated by spectrally and spatially resolved X-ray images (Vink et al. 1999; Holt et al. 1994; Hughes et al. 2000). Even in this case, the optimum angular cut for a point source would reject less than $5 \%$ more $\gamma$ 's than expected. Therefore, deviating from the preliminary analysis presented in Pühlhofer et al. (1999b), we treat Cas $\mathrm{A}$ as a point source.

Figure 5 shows the position of Cas A, reconstructed from $\mathrm{TeV} \gamma$-rays, and superimposed on the high resolution Chandra X-ray image (Hughes et al. 2000). Pointing calibration and source reconstruction uses the methods described by Pühlhofer et al. (1997). The source position is determined by a fit of a 2-dimensional Gaussian to the measured events in celestial coordinates. In order to increase the signal-to-noise ratio, only 3- and 4-telescope events of the full data sample were used (see Sect. 3.3). We conclude that within statistical errors, the TeV emission is centered on the source, and extended emission can neither be proven nor be excluded.

\section{Implications for cosmic ray acceleration}

The detection of $\mathrm{TeV} \gamma$-rays proves that Cas $\mathrm{A}$ is a site of CR acceleration for particles - either nucleons or electrons - with multi-TeV energies. Since it is a shell-type remnant, this detection adds further support to the theory of SNRs being responsible for CR acceleration via the shock acceleration mechanism. However, further conclusions rely on the identification of the hadronic and/or leptonic nature of the high energy primary particles.

Considerable effort has gone into the understanding of the primary electron population, which can be traced by its synchrotron radiation at various wavebands, from radio to X-rays. Especially the hard X-ray spectrum has been interpreted as stand-alone proof for $40 \mathrm{TeV}$ electrons (Allen et al. 1997). A source model has been developed to predict the $\mathrm{TeV}$ emission in the framework of a multi-wavelength study (Atoyan et al. 2000a). The basic challenge is the electron transport in the highly non-homogenous source. The variations in the prediction for $\mathrm{TeV}$ emission via the inverse Compton (IC) mechanism, which will dominate the emission above $1 \mathrm{TeV}$, are mainly due to different deduced electron spectra; the target photon density - the synchrotron photons, the thermal dust emission in the far infrared, the optical/IR line photons, and the microwave background radiation - is well-known. X-ray emission, on the other hand, traces both the electrons and the magnetic field strength, which is typically derived to be of the order of milli-Gauss. The high energy X-ray tail and the TeV flux are spatially unresolved, and hence could be emitted in different regions with different electron spectra.

The electron transport model which attempts to take the spatial structure of Cas A into account predicts a range 
of possible fluxes (Atoyan et al. 2000b). Interestingly, the shape of the spectrum at $\mathrm{TeV}$ energies remains constant, and shows a steep cutoff with a differential spectral index of below -3 above $1 \mathrm{TeV}$. If this holds true for all possible scenarios, it offers a chance to discriminate leptonically induced $\gamma$-rays from the hadronic channel.

The acceleration of hadronic CR in the shock of a SNR is theoretically fairly well-understood for the case of expansion into a homogenous interstellar medium (Berezhko \& Völk 1997, 2000b; Aharonian et al. 2000c). Strong modifications are expected if the shock expands into the wind structure, e.g. of a Wolf-Rayet progenitor star (Berezhko $\&$ Völk 2000a). These calculations show that high $\pi^{0}$ decay $\gamma$-ray fluxes can be expected even for young SNRs like Cas A. However, the influence of the magnetic field configuration in progenitor winds may result in largely perpendicular shocks, with strongly reduced injection efficiency, which would lower the expected $\gamma$-ray fluxes considerably (Völk 1997).

Given these arguments, the expected absolute flux level of $\pi^{0}$-decay $\gamma$-rays is presently not well determined. The value assumed in Atoyan et al. (2000b) and used later in the comparison has been chosen such that the total energy of relativistic protons is $20 \%$ of the CR energy sum of about $10^{50} \mathrm{ergs}$, which is ultimately released by an average Galactic SNR. This reasonable choice does not violate the upper limits from the EGRET detector as well as from Whipple (Lessard et al. 1999) and CAT (Goret et al. 1999) at $\mathrm{TeV}$ energies. Since it cannot be increased substantially without doing so, it can be considered as a model upper limit. Again, the shape of the spectrum remains as a common feature, the $\pi^{0}$-decay spectrum should extend up to $1 \mathrm{TeV}$ with a hard $E^{-2.1 \ldots 2.2}$ power-law.

Figure 6 shows the measured $\mathrm{TeV}$ flux of Cas A, together with the $1 \sigma$ error band of the spectral index under the assumption of a power-law spectrum between 1 and $10 \mathrm{TeV}$. The TeV upper limits are taken from Lessard et al. (1999) and Goret et al. (1999). Indicated are the expected spectra for IC plus bremsstrahlung emission, for different model parameters (solid and dashed lines) as described in Atoyan et al. (2000b). The dotted curve shows the predicted spectrum for the $\pi^{0}$-decay $\gamma$-ray flux.

Slope: The slope of the spectrum marginally favours hadronic emission, but the data are also well compatible with the leptonic spectrum; the respective chance probabilities are $98 \%$ and $15 \%$ (for details see Sect. 3.5). Moreover, the discrepancies between data and leptonic models might also be attributable to the complexity of the source and the resulting possible model variations.

Integral flux: Given the uncertainties in the acceleration efficiency and the resulting uncertainty of the absolute $\pi^{0}$ decay flux as discussed above, the measured flux is consistent with hadronic emission; it is also in good agreement with one leptonic prediction.

In summary, our results may at present be interpreted as leptonic or hadronic emission (or a mixture of both). It remains to be seen whether the results of future theoretical work using detailed acceleration models will allow definite

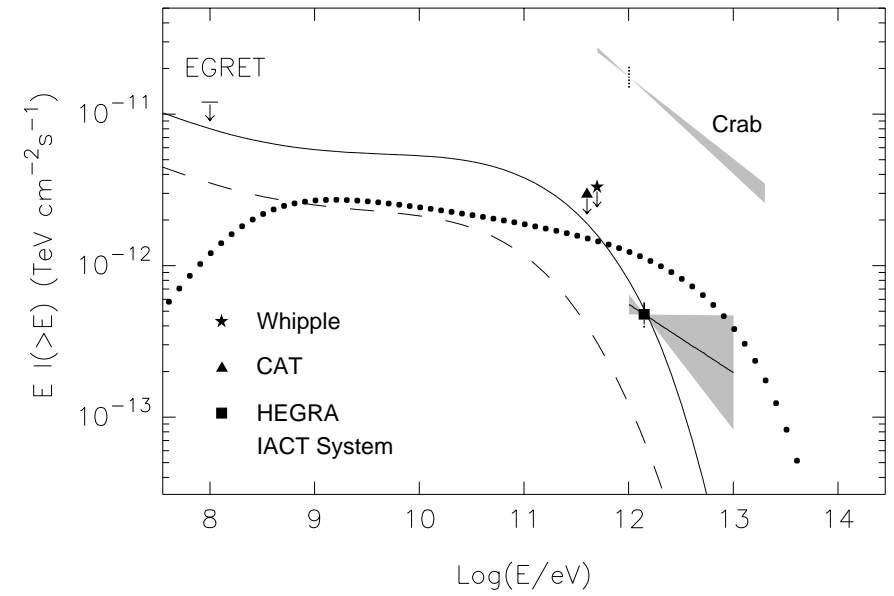

Fig. 6. The measured flux and spectral index of Cas A in the context of model predictions. The shaded area shows the $1 \sigma$ error range for the measured spectral distribution under the assumption of a $E^{-\alpha}$ power law spectrum. The dotted curve represents the model spectrum for the $\pi^{0}$-decay flux as discussed in the text; the current model however allows a renormalisation of the spectrum. The solid and dashed lines show the predicted IC plus bremsstrahlung flux for different model parameters; see Atoyan et al. (2000b) for details. Also indicated are the upper limits measured by EGRET, Whipple and CAT

conclusions about the nature of the primary $\mathrm{CR}$ particles which lead to the $\mathrm{TeV}$ emission from Cas A.

Acknowledgements. The support of the German ministry for Research and technology BMBF and of the Spanish Research Council CICYT is gratefully acknowledged. G. P. R. acknowledges receipt of a Humboldt fellowship. We thank the Instituto de Astrofísica de Canarias for the use of the site and for supplying excellent working conditions at La Palma. We gratefully acknowledge the technical support staff of the Heidelberg, Kiel, Munich, and Yerevan Institutes.

\section{References}

Aharonian, F. A., Akhperjanian, A. G., Barrio, J. A., et al. 1999, A\&A, 342, 69

Aharonian, F. A., Akhperjanian, A. G., Barrio, J. A., et al. 2000a, ApJ, 539, 317

Aharonian, F. A., Akhperjanian, A. G., Barrio, J. A., et al. 2000b, A\&A, 361, 1073

Aharonian, F. A., Akhperjanian, A. G., Barrio, J. A., et al. 2000c, A\&A, submitted

Aharonian, F. A., \& Atoyan, A. M. 1999, A\&A, 351, 330

Alexandreas, D. E., Berley, D., Biller, S., et al. 1993, Nucl. Instr. Meth. A, 328, 570

Allen, G. E., Keohane, J. W., Gotthelf, E. V., et al. 1997, ApJ, 487, L97

Allen, G. E., Gotthelf, E. V., \& Petre, R. 1999, in Proc. of the 26th ICRC, ed. D. Kieda, M. Salamon, \& B. Dingus (Salt Lake City), vol. 3, $480 \mathrm{ff}$

Atoyan, A. M., Tuffs, R. J., Aharonian, F. A., \& Völk, H. J. 2000a, A\&A, 354, 915

Atoyan, A. M., Aharonian, F. A., Tuffs, R. J., \& Völk, H. J. 2000b, A\&A, 355, 211 
Baring, M. G. 2000, in Snowbird Workshop on TeV GammaRay Astronomy, Towards a Major Atmospheric Cherenkov Detector - VI, ed. B. H. Dingus, M. H. Salamon, \& D. B. Kieda (AIP, New York), 173

Berezhko, E. G., Ksenofontov, L. T., \& Petukhov, S. I. 1999, in Proc. of the 26th ICRC, ed. D. Kieda, M. Salamon, \& B. Dingus (Salt Lake City), vol. 4, 431

Berezhko, E. G., \& Völk, H. J. 1997, Astropart. Phys., 3, 183

Berezhko, E. G., \& Völk, H. J. 2000a, A\&A, 357, 283

Berezhko, E. G., \& Völk, H. J. 2000b, Astropart. Phys., 14, 201

Drury, L. O., Aharonian, F. A., \& Völk, H. J. 1994, A\&A, 287, 959

Eadie, W. T., Drijard, D., James, F. E., Roos, M., \& Sadoulet, B. 1971, Statistical Methods in Experimental Physics, North-Holland, Amsterdam

Esposito, J. A., Hunter, S. D., Kanbach, G., \& Sreekumar, P. 1996, ApJ, 461, 820

Favata, F., Vink, J., Fiume, D. D., et al. 1997, A\&A, 324, L49

Fesen, R. A., \& Becker, R. H. 1991, ApJ, 371, 621

Goret, P., Gouiffes, C., Nuss, E., \& Ellison, D. C. 1999, in Proc. of the 26th ICRC, ed. D. Kieda, M. Salamon, \& B. Dingus (Salt Lake City), vol. 3, 496

Heß, M. 1998, Ph.D. Thesis, Ruprecht-Karls-Universität Heidelberg, Heidelberg, Germany

Hofmann, W., Jung, I., Konopelko, A., et al. 1999, Astropart. Phys, 12, 135

Holt, S. S., Gotthelf, E. V., Tsunemi, H., \& Negoro, H. 1994, PASJ, 46, L151

Hughes, J. P., Rakowski, C. E., Burrows, D. N., \& Slane, P. O. 2000, ApJ, 528, L109

Iyudin, A. F., Diehl, R., Lichti, G. G., et al. 1997, in 2nd INTEGRAL Workshop The Transparent Universe, St. Malo, France (ESA Publications Division, ESTEC, Noordwijk,
The Netherlands), 37

Konopelko, A., Aharonian, F., Hemberger, M., et al. 1999a, J. Phys. G, 25, 1989

Konopelko, A., Hemberger, M., Aharonian, F., et al. 1999b, Astropart. Phys., 10, 275

Koyama, K., Petre, R., Gotthelf, E. V., et al. 1995, Nature, 378,255

Koyama, K., Kinugasa, K., Matsuzaki, K., et al. 1997, PASJ, 49, L7

Lessard, R. W., Bond, I. H., Boyle, P. J., et al. 1999, in Proc. of the 26th ICRC, ed. D. Kieda, M. Salamon, \& B. Dingus (Salt Lake City), vol. 3, 488

Li, T.-P., \& Ma, Y.-Q. 1983, ApJ, 272, 317

Muraishi, H., Tanimori, T., Yanagita, S., et al. 2000, A\&A, 345, L57

Pühlhofer, G., Daum, A., Hermann, G., et al. 1997, Astropart. Phys., 8, 101

Pühlhofer, G., Bernlöhr, K., \& Daum, A. 1999a, in Proc. of the 26th ICRC, ed. D. Kieda, M. Salamon, \& B. Dingus (Salt Lake City), vol. 4, 77

Pühlhofer, G., Völk, H., \& Wiedner, C. A. 1999b, in Proc. of the 26 th ICRC, ed. D. Kieda, M. Salamon, \& B. Dingus (Salt Lake City), vol. 3, 492

Reed, J. E., Hester, J. J., Fabian, A. C., \& Winkler, P. F. 1995, ApJ, 440, 706

Tanimori, T., et al. 1998, ApJ, 497, L25

Vink, J., Maccarone, M. C., Kaastra, J. S., et al. 1999, A\&A, 344,289

Völk, H. J. 1997, in Kruger National Park Workshop on TeV Gamma-Ray Astronomy, Towards a Major Atmospheric Cherenkov Detector - V, 87, Space Reasearch Unit, WESTPRINT-Potchefstroom, South Africa, ed. O. C. de Jager 